\title{
Antimicrobial therapy in neonatal intensive care unit
}

\author{
Chryssoula Tzialla ${ }^{1 *}$, Alessandro Borghesi ${ }^{1}$, Gregorio Serra ${ }^{2}$, Mauro Stronati ${ }^{1}$ and Giovanni Corsello ${ }^{2}$
}

\begin{abstract}
Severe infections represent the main cause of neonatal mortality accounting for more than one million neonatal deaths worldwide every year. Antibiotics are the most commonly prescribed medications in neonatal intensive care units (NICUs) and in industrialized countries about $1 \%$ of neonates are exposed to antibiotic therapy. Sepsis has often nonspecific signs and symptoms and empiric antimicrobial therapy is promptly initiated in high risk of sepsis or symptomatic infants. However continued use of empiric broad-spectrum antibiotic treatment in the setting of negative cultures especially in preterm infants may not be harmless.

The benefits of antibiotic therapy when indicated are clearly enormous, but the continued use of antibiotics without any microbiological justification is dangerous and only leads to adverse events. The purpose of this review is to highlight the inappropriate use of antibiotics in the NICUs, to exam the impact of antibiotic treatment in preterm infants with negative cultures and to summarize existing knowledge regarding the appropriate choice of antimicrobial agents and optimal duration of therapy in neonates with suspected or culture-proven sepsis in order to prevent serious consequences.
\end{abstract}

Keywords: Newborn, Antibiotic, Neonatal sepsis, Resistant bacteria, Empiric therapy, Antibiotic stewardship

\section{Introduction}

Sepsis represent the main cause of neonatal mortality accounting for more than one million neonatal deaths worldwide every year, and antibiotics are the most commonly prescribed medications in the neonatal intensive care units (NICU) [1,2]. Sepsis has often nonspecific signs and implies in serious consequences; as a result, empirical antimicrobial therapy is promptly initiated in symptomatic infants with suspected sepsis after obtaining biological material for culture [1]. However, neonates who do not have infection often receive antimicrobial agents during hospital stay, and inappropriate empirical antibiotic treatment may have serious side effects [3].

Nearly all extremely low birth weight infants (ELBW) infants admitted to a NICU receive an empirical antibiotic treatment in the first postnatal days, in spite of sterile cultures and low incidence of culture-proven bacterial sepsis in this population $[1,3,4]$. This observation has been confirmed by a study of the National Institute

\footnotetext{
* Correspondence: c.tzialla@smatteo.pv.it

${ }^{1}$ Neonatologia, Patologia Neonatale e Terapia Intensiva neonatale,

Fondazione IRCCS Policlinico San Matteo, Piazzale Golgi 19, 27100 Pavia, Italy Full list of author information is available at the end of the article
}

of Child Health and Human Development National Research Network on 6956 very low birth weight (VLBW) infants, showing that $56 \%$ of all infants received at least one course of antibiotic treatment, even if proven sepsis was diagnosed in only $21 \%$ of all infants [5].

In this review we describe the use of antibiotics in the NICU, focusing on the potential serious adverse effects of inappropriate use; we identify the opportunities for improving antibiotic prescription in the NICU, and we discuss the future directions of antimicrobial therapy.

\section{Epidemiology of bacterial infections in the NICU}

Neonatal sepsis can be classified as early-onset (EOS) and late-onset (LOS) sepsis. EOS is most often caused by group B streptococcus (GBS) (43\%), followed by Escherichia coli (15.5-29\%). Among VLBW infants, the rate of Escherichia coli infection exceeds that of GBS infection (5.1 vs 2.1 per 1000 live births) [3].

LOS is mainly caused by Gram-positive bacteria (GPB) (49\%), most often coagulase-negative Staphylococcus (CoNS) (45\%). Gram-negative LOS is less common (23\%), but is associated with greater mortality in the NICU (19$36 \%)[6,7]$. 
As showed by various studies, antibiotics such as ampicillin, gentamicin and cefotaxime commonly used for empirical therapy appear to be appropriate. In a recent study, investigators revealed that more than $94 \%$ of the EOS isolates were susceptible to penicillin and gentamicin, to amoxicillin and cefotaxime and to cefotaxime alone. The LOS isolates (excluding CONS) had a more than $96 \%$ susceptibility to flucloxacillin or amoxicillin and gentamicin, to amoxicillin and cefotaxime, but only $78 \%$ to cefotaxime alone. The investigators concluded that cefotaxime should not be included in the empiric regimen of suspected sepsis, because of lower susceptibility levels [7].

Blackburn et $a l$, in a study of neonatal septicemia found that only $1.4 \%$ of Gram-negative bacteria (GNB) were resistant to penicillin plus gentamicin, whereas $10.4 \%$ of isolates tested against amoxicillin plus cefotaxime were resistant to this association [8].

Most hospital acquired CoNS are resistant to many commonly prescribed antibiotics. In the NICU, enterococci are less frequently isolated than staphylococcal species. Nevertheless, ampicillin-resistant, and, more recently, vancomycin-resistant enterococci have been described and have become endemic in some NICUs [9].

GNB are often resistant to at least one class of antibiotics usually used, and bacteria that are multi- or extensivelyresistant to conventional antibiotics are frequently isolated. Pan-resistant pathogens are rarely isolated in the NICUs, where resistance is most frequently found to piperacillintazobactam, ceftazidime, and/or gentamicin $[9,10]$.

Moreover, the emergence of extended-spectrum $\beta$-lactamase (ESBL)-producing GNB confers resistance to penicillins and cephalosporins, often coexisting with resistance to other antibiotic categories as fluoroquinolones and aminoglycosides $[9,11]$.

\section{Risks associated with empirical administration of broad-spectrum antibiotics}

The use of broad-spectrum antibiotics is associated with different adverse effects: alteration of gut colonization, emergency of resistant strains, and increasing risk of Candida colonization and subsequent invasive candidiasis [4].

All antibiotics can alter gut colonization of the patient, promoting either antibiotic resistance among normal commensal organisms or the emergence of other pathogens $[4,12]$.

A number of in vitro and in vivo studies have shown that, although short courses of carbapanems and thirdgeneration cephalosporins cover a broad spectrum of bacteria, their prolonged and intensive use selects resistant bacteria. Overuse of third-generation cephalosporins favors the emergence of ESBL-producing strains of GNB in NICUs $[10,13]$.
In order to study the effects of empirical antibiotics on the emergence of resistant pathogens, de Man et al. [14] examined 436 infants admitted to 2 NICUs who were assigned initially to either a narrow-spectrum antibiotic regimen (penicillin or flucloxacillin plus tobramycin) or a broad-spectrum regimen (amoxicillin plus cefotaxime) and exchanged regimens after 6 months. The investigators demonstrated that the relative risk for colonization with strains resistant to empirical therapy per 1000 patients at risk was 18-fold higher in the broad-spectrum regimen group than in the narrow-spectrum regimen group.

Exposure to broad spectrum antibiotics has been also associated with the emergence of invasive candidiasis. In a cohort of 3,702 ELBW infants, previous use of third generation cephalosporins or carbapenems were associated with an increased risk of invasive candidiasis (OR 2.2, 95\% CI 1.4-3.3). The incidence of candidiasis between centers varied from $2.4 \%$ to $20.2 \%$ and correlated with the average number of days of broad spectrum antibiotic use per infant with sterile cultures throughout hospitalization [15].

A multicenter cohort study of 128,914 neonates, revealed that the use of ampicillin/cefotaxime during the first 3 days after birth is associated with an increased risk of death before discharge (OR 1.5, 95\% CI 1.4-1.7) compared with the use of ampicillin/gentamicin, even if the authors highlighted that this observation may be limited by selection bias. The authors concluded that, for patients receiving ampicillin, the concurrent use of cefotaxime during the first three days after birth is either a surrogate for an unrecognized factor or is itself associated with an increased risk of death, compared with the concurrent use of gentamicin [1].

\section{Adverse effects of prolonged courses of empirical antibiotic treatment}

For culture-proven sepsis a full course of antibiotics is indicated. Conversely, concerns remain about the optimal length of antibiotic therapy for clinical, not microbiologically demonstrated sepsis. Recent cohort studies show an association between the duration of empirical antibiotic therapy and mortality, necrotizing enterocolitis (NEC) and LOS.

Cotten et al. [16] conducted a retrospective cohort analysis of 5,693 ELBW infants admitted to 19 tertiary centers. Of 5,693 infants, 4,039 survived $>5$ days, received initial empirical antibiotic treatment and had sterile initial blood culture at 72 hours of life. In a multivariate analysis adjusted for risk factors, prolonged duration of therapy was associated with increased odds of NEC or death or death alone. Each additional day of antibiotic therapy was associated with a $4 \%$ increase in the odds of NEC or death, 
a $7 \%$ increase in the odds of NEC alone and a $16 \%$ increase in the odds of death alone.

A retrospective 2:1 control case analysis examined the association between antibiotic exposure and the risk of NEC. When neonates with sepsis were removed from cohort, antibiotic duration increased the risk of NEC by approximately $20 \%$ per day of exposure $(\mathrm{OR}=1.2)$. Exposure for $>10$ days resulted in nearly a threefold increase in the risk of developing NEC [17].

Prolonged antibiotic therapy has also been associated with LOS. A retrospective study of 365 infants $\leq 32$ weeks gestational age (GA) and $\leq 1500 \mathrm{~g}$ birth weight (BW), who survived free of sepsis and NEC in the first week of life, found that prolonged antibiotic therapy ( $\geq 5$ days) initiated on the day of birth was independently associated with LOS alone and the composite outcome of LOS, NEC or death. Each additional day of antibiotics was associated with a significantly increased risk of these outcomes. For infants who received any initial empirical antibiotic exposure, the adjusted attributable risk for LOS, NEC or death was $32 \%$ and the number needed to harm was 3 [18].

\section{Recommendations for a judicious use of antibiotics Choice of the antibiotic agent}

Concerning the newborn infants, there are no randomized controlled trials that can definitely prove the best choice of antibiotics. However, many authors agree that an association of a penicillin or semisynthetic penicillin (ampicillin) together with an aminoglycoside is effective against microorganisms causing EOS, and therefore can be considered the best empirical regimen $[10,19,20]$.

For the treatment of suspected LOS, different authors agree that the best regimen is an antistaphylococcal penicillin (oxacillin, flucloxacillin) together with an aminoglycoside; the choice of vancomycin should be restricted to microbiologically demonstrated cases of methicillinresistant Staphylococcus aureus (MRSA) or CoNS [19,20].

In a recent review, Sivanandan et al. [21] recommended the same antibiotic combinations for empirical therapy of EOS and LOS in neonates. In case of LOS in an instable neonate and in areas where MRSA is prevalent, vancomycin and a third-generation cephalosporin should be considered. For the treatment of suspected early-onset meningitis the authors recommended a combination of ampicillin and aminoglycoside or ampicillin and cefotaxime, and in case of late-onset meningitis a combination of an antistaphilococcal antibiotic (nafcillin or vancomycin) plus a third-generation cephalosporin with or without aminoglycoside.

Russel et al. [12] for the therapy of meningitis suggest as first line treatment the combination of cefotaxime and amoxicillin with or without gentamicin.
Different authors state that empirical therapy should never be started with a broad spectrum antibiotic such as a third-generation cephalosporin or a carbapenem, and their use should be restricted to particular cases $[13,19]$. Gray et al. [10] suggest the use of piperacillin-tazobactam in the units where aminoglycoside-resistant $\mathrm{GBN}$ are prevalent as an alternative to third generation cephalosporins.

Russel et al. in a recent review, based on epidemiological data from UK neonatal infection surveillance studies, suggest for EOS and LOS treatment, antibiotic strategies reported in Table 1 [12].

\section{Duration of the antibiotic course}

Culture-proven neonatal sepsis is treated with full course of appropriate antibiotics; the appropriate duration of the antibiotic course is more difficult to be established in case of suspected (clinical) sepsis with negative cultures. Usually, antibiotics are discontinued as soon as blood cultures are confirmed negative (48-72 hours), and if laboratory

\section{Table 1 Choice of antibiotics}

\begin{tabular}{ll}
\hline EOS & Penicillin + gentamicin \\
& - if Listeria monocytogenes: amoxicillin + \\
& gentamicin \\
& - if S.aureus: flucloxacillin + gentamicin \\
& First line: flucloxacillin + gentamicin \\
& Second line: \\
& - vancomycin + gentamicin (with caution) \\
& - vancomycin + piperacillin/tazobactam \\
& (to extend Gram-negative cover) \\
& Third line: meropenem, ciprofloxacin \\
& First line: cefotaxime with amoxicillin \pm \\
& gentamicin \\
Meningitis & Second line: meropenem
\end{tabular}

Gram positive Currently: glycopeptide antibiotics are the multiresistant bacteria mainstay of therapy, especially vancomycin; if necessary linezolid, clindamycin, rifampicin and daptomycin could be alternative regimens

In the future: novel cephalosporins like ceftaroline and ceftobiprole; novel lipoglycopeptide antibiotics are oritavacin and dalbavancin; telavacin has been approved in the USA in adults

$\begin{array}{ll}\text { Gram negative } & \text { Currently: aminoglycosides and } \\ \text { multiresistant bacteria } & \text { cephalosporins are the antibiotics of choice; } \\ \text { carbapenems, colistin, co-trimoxazole, } \\ \text { ticarcillin-clavulanic acid could be the an } \\ \text { alternative; fluoroquinolone, ciprofloxacin, } \\ \text { tigecycline and tetraciclins could only be } \\ \text { justified in extreme cases. } \\ \text { In the future: treatment options are } \\ \text { extremely limited }\end{array}$

Modified from: Russell AB, Sharland M, Heath PT. Improving antibiotic prescribing in neonatal units: time to act. Arch Dis Fetal Neonatal 2012; 97:F141-146 and Gray JW, Patel M. Management of antibiotic-resistant infection in the newborn. Arch Dis Child Educ Pract 2011 Aug;96(4):122-7. 
results and the evolution of the clinical signs allow to exclude an infection [15,21-24].

In case of abnormal laboratory tests [white blood cell count and C-reactive protein (CRP) at age 6-12 h] in well-appearing neonate with negative blood culture Polin and the Committee on Fetus and Newborn [11] (COFN) suggest to continue empiric antibiotic therapy if mother received antibiotics during labor and delivery in case of infants $<37$ weeks' gestation with risk factors for sepsis and infants $\geq 37$ weeks' gestation born from mothers with chorioamnionitis.

The algorithms for duration of empiric therapy when cultures are sterile suggested by COFN generated discussion about the lack of strong supportive evidence to guide decisions to stop antimicrobials at $48 \mathrm{~h}$ in certain cases.

Cotten et al. [25] in a recent review highlight that current studies are inadequate to specify appropriate testing a timing of diagnostic tests in all situations in which empirical therapy have been started. The authors offer the following suggestions for the management of term and late preterm neonates on empirical therapy for EOS with negative cultures at 48 postnatal hours: i) continuation of treatment for 7 days if clinical signs of sepsis persist over 24 hours; ii) stop antibiotics at 48 hours in asymptomatic neonates with initial (4 postnatal hours) normal complete blood count (initial laboratory tests drawn by risk factors), and in neonates with transient clinical signs (lasting less than $24 \mathrm{~h}$ ), and abnormal initial complete blood count, if serial CRP measurements at 24 and 48 hours are low in a well-appearing neonate.

In case of culture-proven sepsis, Sivanandan et al. [21] suggest that it is reasonable to treat for 10-14 days with appropriate antimicrobial agents infants with blood culture-proven sepsis without meningitis. However, in selected situations [i.e. neonates $>32$ weeks GA and $>1500 \mathrm{~g}$ $\mathrm{BW}$, who became asymptomatic with 5 days of appropriate therapy], it is reasonable to discontinue antibiotics at 7-10 days if laboratory results are normal and cultures are sterile in a well-appearing child. For neonatal meningitis, the same authors suggest a duration of therapy of 14 to 21 days for GBS, $\geq 21$ days for Lysteria monocytogenes, a minimum of 21 days for Gram-negative meningitis and 4 to 6 weeks in cases complicated with intracranial abscesses.

\section{Therapies for resistant pathogens}

Even though an increase in vancomycin MIC values, within the susceptible range, has been registered among isolates of MRSA, CoNS or S. aureus strains vancomycinintermediate or vancomycin-resistant have not been isolated from a NICU population so far. Consequently, glycopeptides remain an appropriate treatment for most staphylococcal infections in this setting $[9,26]$. However in case of unresponsive Gram-positive infections, linezolid has been the most used in neonatology, even if the use of daptomycin have been described in few reports in case of persistent staphylococcal bacteremia in neonates [26].

Several novel antibiotics active against GPB are currently in diverse phases of development and clinical trials are ongoing. In particular, advanced-generation cephalosporins like ceftaroline and ceftobiprole with activity against multidrugresistant staphylococci have been reported in adults $[26,27]$, as well as lipoglycopeptides agents with activity against multidrug-resistant gram-positive pathogens like oritavancin and dalbavancin and telavancin. All three agents are promising alternatives for the treatment of complicated skin and soft-tissue infections in adults but there are no data about their pharmacokinetics in neonates $[26,28]$.

For serious antibiotic-resistant GNB infections, carbapenems have become the mainstay of treatment with meropenem being the most widely used and doripenem as a newer carbapenem with greater activity against Pseudomonas aeruginosa [10]. However rapid emergence of resistance to these antibiotics means that the use of agents such as colistin, fosfomycin and tigecycline must be considered. Colistin is largely experienced in the neonatal population, but it must be kept in mind that is not effective against Proteus and Serratia. There is little experience of using fosfomycin in neonates but is worth considering as a final-resort therapy for extensively drug-resistant GNB $[10,26]$. Tigecycline, active against hard to treat pathogens like many multidrug-resistant GPB e GNB, is inactive against Pseudomonas Aeruginosa [10,29], but due to the possible effects on the bone growth in children, the use in neonates could only be justified in extreme cases (Table 1) $[10,26]$.

\section{Future strategies}

\section{Appropriate antibiotic policies}

Antimicrobial stewardship programs (ASP) were introduced in the 1980s, with the aim to reduce unnecessary therapies. Nevertheless, only in 2007, the Infectious Diseases Society of America, together with other professional organizations, published guidelines in order to implement multidisciplinary ASP [30]. However, in spite of positive experiences on adults, data about the consequences of ASPs in neonatal settings are lacking.

Recently, several authors suggested different strategies that might be helpful in a NICU that include implementation of systems for surveillance of bloodstream infections, education of practitioners concerning the development of resistance, use of narrow spectrum empirical antibiotic policy and stop of empirical treatment or documented justification for continuation when blood cultures are negative, use of narrowest spectrum antibiotics for a proven infection, formulary restriction and pre-authorization requirements for selected antimicrobial agents like cephalosporins, meropenem, vancomycin and teicoplanin [11,12]. 
Patel et al. [31] suggest that although specific guidelines for neonates are often lacking, antibiotic stewardship principles like those proposed by the Get Smart for Health Care Campaign of the Centers for Disease Control and Prevention can be applied to the NICU along with the development of an interdisciplinary antimicrobial stewardship team and metrics to measure successful implementation of ASP.

\section{Development of innovative treatments}

Spellberg et al. [32].suggest future strategies to combat antibiotic-resistance like therapies with diminished potential to drive resistance (pe infusion of monoclonal antibodies and white cells that kill microbes or biologic agents that alter bacterial ability to trigger inflammation) and treatments that alter host-microbe interactions like moderation of host inflammation and limitation of microbial growth (pe sequestration of host nutrients, probiotics administration that compete with microbial growth).

\section{Discovery of new antibiotics}

The discovery of new antibiotics must face a number of challenges that make the development of new antibiotic drugs more difficult compared to other non-antibiotic drugs. These have been well summarized in a recent review by Lewis et al. [33].

First, the poor penetration of antibiotics in prokaryotic cells requires the delivery of higher amounts of a compound which, in turn, increases the risk of toxicity and narrows the therapeutic range.

In addition, specifically targeting GNB is even more challenging, as not only the inner membrane restricts the penetration of hydrophilic substances, but also the outer membrane further reduces the number of compounds that may be effective, and the multidrug-resistant pumps extrude any compounds that leak in through the outer membrane.

Even when these pharmacodynamics-related issues are resolved, the development of a new drug needs to face the pharmacokinetics-related issues; indeed, the search for molecules with physicochemical properties to improve the likelihood of bioavailability (e.g. by applying the Lipinski's rules) may not match with the need of a compound with physicochemical properties that improve penetration into prokaryotes.

Once discovered, the compound must be tested in clinical trials; however, the identification and recruitment of patients infected with multi-resistant bacteria may be difficult, as most infections are caused by pathogens susceptible to the available compounds.

Finally, there is modest return on investment on antibiotic development compared to other drugs. Indeed, antibiotic therapy is typically short-term, lasting only some days, while therapies with cholesterol-lowering drugs or with anti-hypertensive drugs last for years, or lifelong; and, in any case, resistance to the new antibiotics will eventually develop, limiting their use and the profits that it produces.

Despite all these considerations, most of the potential bacterial targets for antibiotics are still unexploited. It is assumed that there are approximately 200 conserved essential proteins in bacteria, but the current antibiotics only hit few targets or pathways [33]. Future efforts should focus on the discovery of compounds directed against these new targets.

\section{Conclusions}

Sepsis represents the main cause of neonatal mortality and antibiotics are the most commonly prescribed medications in the NICUs. Wise choice of antimicrobial agents and optimal duration of therapy in neonates with suspected or culture-proven sepsis is crucial in order to limit the use of unnecessary broad spectrum antibiotic therapy, and to provide local solutions to the world-wide race against antimicrobial resistance. While there is an increasing choice of drugs for treating multiresistant GPB, alternatives for GNB will be seriously restricted for the foreseeable future. This makes important the need to promote attitudes that fortify measures for infection-control and to develop new treatments that complement traditional approaches.

\section{Abbreviations}

NICU: Neonatal intensive care units; ELBW: Extremely low birth weight infants; VLBW: Very low birth weight; EOS: Early-onset sepsis; LOS: Late-onset sepsis; GBS: Group B streptococcus; CoNS: Coagulase-negative Staphylococcus; GPB: Gram-positive bacteria; GNB: Gram-negative bacteria; ESBL: Extendedspectrum $\beta$-lactamase; NEC: Necrotizing enterocolitis; GA: Gestational age; BW: Birth weight; MRSA: Methicillin-resistant Staphylococcus aureus; CRP: Creactive protein; COFN: Committee on Fetus and Newborn; ASP: Antimicrobial stewardship programs.

\section{Competing interests}

The authors declare that they have no competing interests.

\section{Authors' contributions}

$C T$ reviewed the relevant articles on the literature and drew the first draft; $A B$ have been involved in revising the English version of the manuscript; GS reviewed the relevant articles on the literature under the supervision of CT; MS have made substantial contributions to conception and design and critically revised the final draft for important intellectual content; GC critically revised the final draft for important intellectual content. All authors read and approved the final manuscript.

\section{Author details \\ ${ }^{1}$ Neonatologia, Patologia Neonatale e Terapia Intensiva neonatale, Fondazione IRCCS Policlinico San Matteo, Piazzale Golgi 19, 27100 Pavia, Italy. ${ }^{2}$ Terapia Intensiva Neonatale Dipartimento di Scienze per la Promozione della Salute e Materno Infantile "G. D'Alessandro", Università degli Studi di Palermo, Via Alfonso Giordano, 390127 Palermo, Italy.}

Received: 22 August 2014 Accepted: 4 February 2015

Published online: 01 April 2015

\section{References}

1. Clark RH, Bloom BT, Spitzer AR, Gerstmann DR. Empiric use of ampicillin and cefotaxime, compared with ampicillin and gentamicin, for neonates at risk for sepsis is associated with an increased risk of neonatal death. Pediatrics. 2006;117(1):67-74. 
2. Depani SJ, Ladhani S, Heath PT, Lamagni TL, Johnson AP, Pebody RG, et al. The contribution of infections to neonatal deaths in England and Wales. Pediatr Infect Dis J. 2011;30(4):345-7.

3. Stoll BJ, Hansen NI, Sánchez PG, Faix RG, Poindexter BB, Van Meurs KP, et al. Early onset neonatal sepsis: the burden of group B Streptococcal and E. coli disease continues. Pediatrics. 2011;127(5):817-26.

4. Tripathi N, Cotton CM, Smith PB. Antibiotic use and misuse in the neonatal intensive care unit. Clin Perinatol. 2012;39(1):61-8.

5. Stoll BJ, Hansen N, Fanaroff AA, Wright LL, Carlo WA, Ehrenkranz RA, et al. Late onset sepsis in very low birth weight neonates: the experience of the NICHD Neonatal Research Network. Pediatrics. 2002;110(2):285-91.

6. Vergnano S, Menson E, Kennea N, Embleton N, Russell AB, Watts T, et al. Neonatal infections in England: the NeonIN surveillance network. Arch Dis Child Fetal Neonatal Ed. 2011;96:F9-14.

7. Muller-Pebody B, Johnson AP, Heath PT, Gilbert R, Henderson KL, Sharland M. iCAP Group (Improving Antibiotic Prescribing in Primary Care.) Empirica treatment of neonatal sepsis: are the current quidelines adequate? Arch Dis Child Fetal Neonatal Ed. 2011;96:F4-8.

8. Blackburn RM, Verlander NQ, Heath PT, Muller-Pebody B. The changing antibiotic susceptibility of bloodstream infections in the first month of life: informing antibiotic policies for early- and late-onset neonatal sepsis. Epidemiol Infect. 2014;142(4):803-11.

9. Patel SJ, Saiman L. Antibiotic Resistance in Neonatal Intensive Care Unit Pathogens: Mechanism, Clinical Impact, and Prevention including Antibiotic Stewardship. Clin Perinatol. 2010;37(3):547-63.

10. Gray JW, Ubhi H, Milner P. Antimicrobial treatments of serious Gram-negative infections in newborns. Curr Infect Dis Rep. 2014;16(2):400-8.

11. Polin RA, Denson S, Brady MT. Committee on Fetus and Newborn, Committee on Infectious Diseases. Strategies for prevention of health care-associated infections in the NICU. Pediatrics. 2012;129(4):e1085-93.

12. Russell $A B$, Sharland M, Heath PT. Improving antibiotic prescribing in neonatal units: time to act. Arch Dis Fetal Neonatal. 2012;97:F141-6.

13. Isaacs D. Unnatural selection: reducing antibiotic resistance in neonatal units. Arch Dis Child Fetal Neonatal Ed. 2006:91(1):F72-4.

14. de Man P, Verhoeven BA, Verbrugh H, Vos MC, van den Anker JN. An antibiotic policy to prevent emergence of resistant bacilli. Lancet. 2000;355(9208):973-8.

15. Cotten CM, McDonald S, Stoll B, Goldberg RN, Poole K, Benjamin Jr DK National Institute for Child Health and Human Development Neonatal Research Network. The association of third-generation cephalosporin use and invasive candidiasis in extremely low birth-weight infants. Pediatrics. 2006;118(2):717-22.

16. Cotten CM, Taylor S, Stoll B, Goldberg RN, Hansen NI, Sánchez PJ, et al. Prolonged duration of initial empirical antibiotic treatment is associated with increased rates of necrotizing enterocolitis and death for extremelylow birth weight infants. Pediatrics. 2009;123:58-66.

17. Alexander VN, Northrup V, Bizzarro MJ. Antibiotic exposure in the newborn intensive care unit and risk of necrotizing enterocolitis. J Pediatr. 2011;159:392-7.

18. Kuppala VS, Meinzen-Derr J, Morrow AL, Schibler KR. Prolonged initial empirical antibiotic treatment is associated with adverse outcomes in premature infants. J Pediatr. 2011;159:720-5.

19. Fanos V, Cuzzolin L, Atzei A, Testa M. Antibiotics and antifungals in neonatal intensive care units: a review. J Chemother. 2007;19(1):5-20.

20. Kaufman D, Fairchild KD. Clinical microbiology of bacterial and fungal sepsis in very-low-birth-weight infants. Clin Microbiol Rev. 2004;17(3):638-80.

21. Sivanandan S, Soraisham AS, Swarnam K. Choice and duration of antimicrobial therapy for neonatal sepsis and meningitis. Int J Pediatr. 2011;2011(712150):1-9.

22. Borghesi A, Stronati M. Strategies for the prevention of hospital-acquired infections in the neonatal intensive care unit. J Hospital Infect. 2008;68(4):293-300.

23. Polin RA. Committee on Fetus and Newborn. Management of neonates with suspected or proven early-onset bacterial sepsis. Pediatrics. 2012;129(5):1006-15.

24. Kaiser JR, Cassat JE, Lewno MJ. Should antibiotics be discontinued after 48 hours for negative late-onset sepsis evaluation in neonatal intensive care unit? J Perinatol. 2002:22:445-7.

25. Cotten CM, Smith PB. Duration of empirical antibiotic therapy for infants suspected of early-onset sepsis. Curr Opin Pediatr. 2013:25(2):167-71.
26. Gray JW, Patel M. Management of antibiotic-resistant infection in the newborn. Arch Dis Child Educ Pract. 2011;96(4):122-7.

27. Bazan JA, Martin SI, Kaye KM. Newer beta-lactam antibiotics: Doripenem, Ceftobiprole, Ceftaroline, and Cefepime. Med Clin N Am. 2011:95:743-60.

28. Stryjewski ME, Barriere SL, O'Riordan W, Dunbar LM, Hopkins A, Genter FC, et al. Efficacy of telavancin in patients with specific types of complicated skin and skin structure infections. J Antimicrob Chemother. 2012;67(6):1496-502.

29. Stein GE, Babinchak T. Tigecycline: an update. Diagn Microbiol Infect Dis. 2013;75(4):331-6.

30. Dellit TH, Owens RC, McGowan Jr JE, Gerding DN, Weinstein RA, Burke JP, et al. Infectious Diseases Society of America; Society for Healthcare Epidemiology of America. Infectious Diseases Society of America and the Society for Healthcare Epidemiology of America guidelines for developing an institutional program to enhance antimicrobial stewardship. Clin Infect Dis. 2007:44(2):159-77.

31. Patel S, Saiman L. Principles and strategies of antimicrobial stewardship in the neonatal intensive care unit. Semin Perinatol. 2012:36:431-6.

32. Spellberg B, Bartlett JG, Gilbert DN. The future of antibiotics and resistance. N EngL J Med. 2013;368(4):299-302.

33. Lewis K. Platforms for antibiotic discovery. Nat Rev Drug Discov. 2013;12(5):371-87.

\section{Submit your next manuscript to BioMed Central and take full advantage of:}

- Convenient online submission

- Thorough peer review

- No space constraints or color figure charges

- Immediate publication on acceptance

- Inclusion in PubMed, CAS, Scopus and Google Scholar

- Research which is freely available for redistribution 